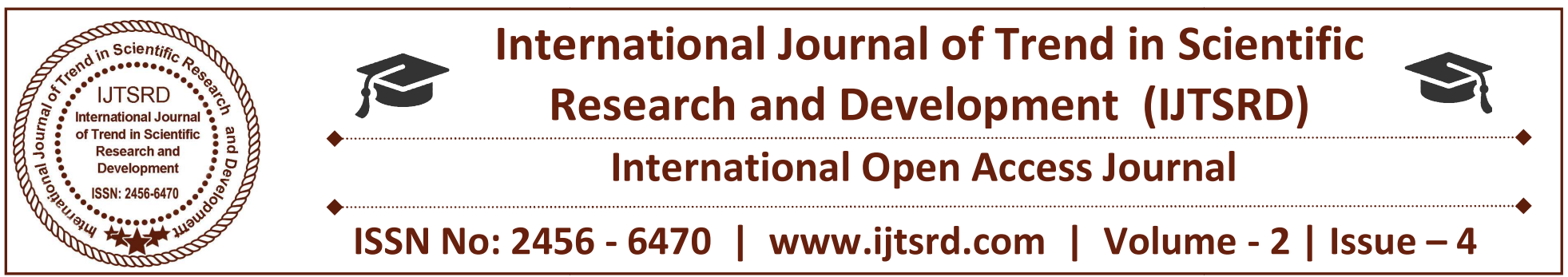

\title{
Personalized Medicine Revolution Medicine based on Genomics Makeup
}

\author{
Ajmal Rasi K P, Puneeth Vishnukeerthy K \\ School of Engineering \& Technology \\ Jain University, Bangalore, Karnataka, India
}

\begin{abstract}
We have acquired the scientific knowledge of medicine based on observation and experience, but it has not always been so. Our ancestors experienced sickness and the fear of death before a rational picture could be made of them, and the medicine of that time was immersed in a system of beliefs, myths and rites. Presently, far too many diseases do not have proven preventions or treatments. There is a dire need to explore into the biological, environmental, and behavioral causal factors of these diseases. Precision medicine is a new science that takes into account the prevention and treatment of diseases by addressing specific changes genetic makeup of each person. Deviating from the conventional "one-size-fits-all" approach, genetic medicine encompasses treatment and prevention strategies for diseases applicable to the average person, and less concerned with differences between individuals.
\end{abstract}

\section{Chapter 1 - Introduction}

The amount of data being digitally collected and stored is vast and expanding rapidly. As a result, the science of data management and analysis is also advancing to enable organizations to convert this vast resource into information and knowledge that helps them achieve their objectives. Computer scientists have invented the term big data to describe this evolving technology. Big data has been successfully used in astronomy, retail sales, search, and politics. Here we will discuss about Health Care.

In the last years it has been born personalized genomics, which tells you your risk factors. This opens a door to personalized medicine, which adjusts treatments to patients depending on their genome. It uses information from a person's genes and proteins to prevent, diagnose and treat a disease, all thanks to the sequencing of the human genome.

The interest in the term genomic medicine has grown lately, partly because drugs are rarely $100 \%$ effective and safe and partly because of developments such as the Human Genome Project. These developments have made it possible to identify subtypes of various diseases on the basis of genetics in addition to other means such as histology, an ability that many believe will lead to an improved capacity to prevent and treat various diseases. For example, knowledge of genetics could help to determine whether patients with certain disease subtypes are more likely than others to be responsive to a particular drug. On the face of it, there seems to be agreement about what genetic medicine entails.

Approximately 99.9 percent of the 3 billion base pairs in an individual's genome are the same as in any other member of the human race. All the differences and features that make someone unique are encoded by only 0.1 percent of their DNA. But 0.1 percent of the genome corresponds to 3 million base pairs, so there is the potential for a lot of genetic differences. Included in these differences are some sixty brand new mutations changes in the sequence of bases in your DNA that have never existed in any person ever before. Everyone really is a mutant. All these genetic 
differences determine not only differences in eye and hair color between someone and anybody else, but also whether anyone have a higher risk of lung cancer or a lower risk of Alzheimer's disease or a greater chance of a heart attack. The ways that someone differ genetically from everybody else also allow the forces of natural selection to work. If an individual prosper and have lots of children that survive, their genetic code will be conserved and passed down to future generations.

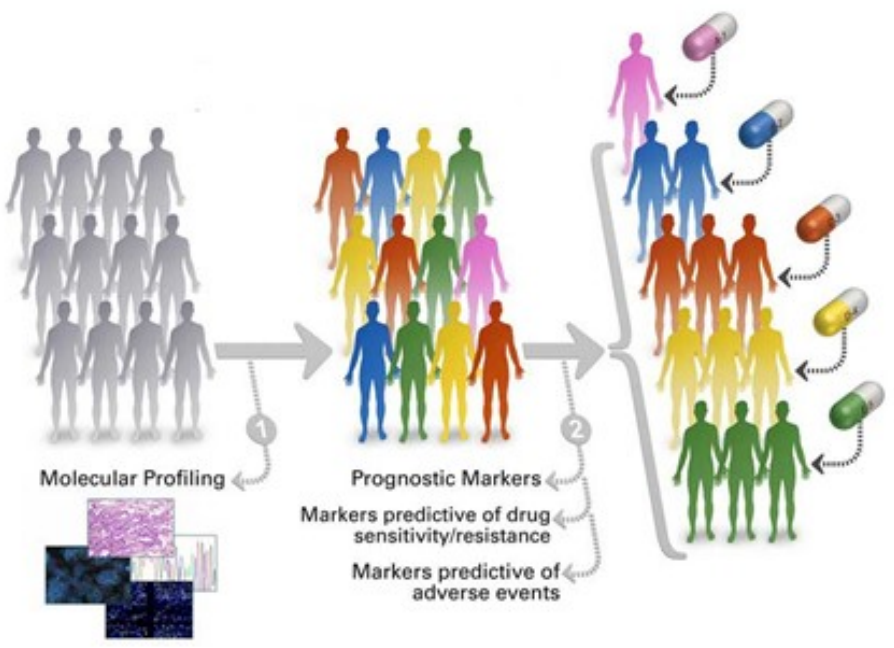

Figure 1 Individualized Medicine

Genomic medicine attempts to build individualized strategies for diagnostic or therapeutic decisionmaking by utilizing patients' genomic information. Big Data analytics uncovers hidden patterns, unknown correlations, and other insights through examining large-scale various data sets. While integration and manipulation of diverse genomic data and comprehensive electronic health records on a Big Data infrastructure exhibit challenges, they also provide a feasible opportunity to develop an efficient and effective approach to identify clinically actionable genetic variants for individualized diagnosis and therapy.

Further examination of the existing definitions of personalized medicine, however, reveals important disparities among them. For example, personalized medicine has been defined as

1. A medical model that proposes the customization of healthcare, with decisions and practices being tailored to the individual patient by use of genetic or other information.

2. The tailoring of medical treatment to the specific characteristics of each patient. It does not literally mean the creation of drugs or medical devices that are unique to a patient. Rather, it involves the ability to classify individuals into subpopulations that are uniquely or disproportionately susceptible to a particular disease or responsive to a specific treatment.

3. A form of medicine that uses information about a person's genes, proteins, and environment to prevent, diagnose, and treat disease.

\section{Chapter 2 - The Existing System}

"One size fits all" is a promise that is rarely kept, whether it is applied to clothing or to medical treatments. Most medical therapies administered to large groups of patients only help a subset of the patients; frequently, we do not know why a particular treatment did not work in a given patient. Indeed, common diseases can have many different causes, and the effectiveness of a particular therapy may depend on the specific disease pathology in an individual patient.

The practice of medicine has always been personal. Doctors use extensive personal information about a patient including medical history, physical exam, vital signs, family history, laboratory measures and imaging tests to determine a patients risk for certain diseases and to make diagnoses.

There is a lot of money spent by pharmaceutical companies and others on advertising the benefits of modern medicine. Consequently, a lot of negative information on the subject does not reach the public domain. Although modern medicine has many advantages and successes, for example, in the treatment of trauma and emergencies, it also has disadvantages and failures.

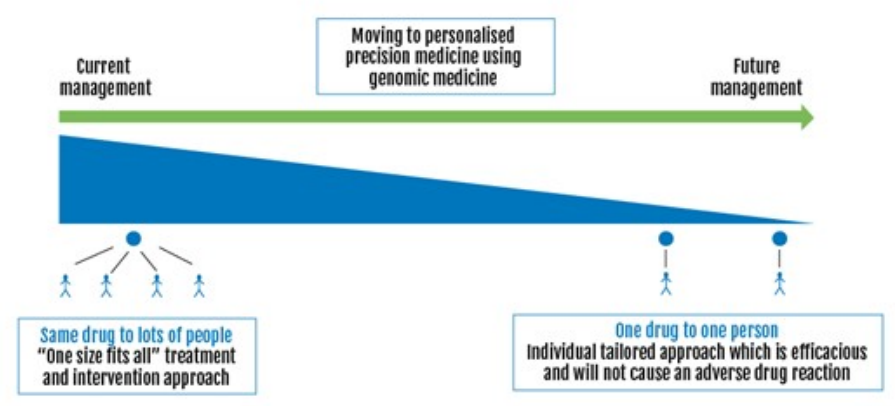

Figure 2 Current One size fit all Approach vs Individualized Approach 
International Journal of Trend in Scientific Research and Development (IJTSRD) ISSN: 2456-6470

\section{Chapter 3 - Limitation of Existing System}

- Many drugs don't work for or harm the patients they are prescribed for due to a "one size fits all" approach.

- Need to determine who will benefit and who will not and avoid "trial and error" therapy.

- Currently disease is often detected too late, leads to high costs and poor outcomes.

- Need to detect and treat signs of disease before it becomes a problem, not after becoming ill.

\section{Drug Efficacy: Less Than $\mathbf{5 0} \%$ of Drugs Work on the Patient They are Prescribed For}

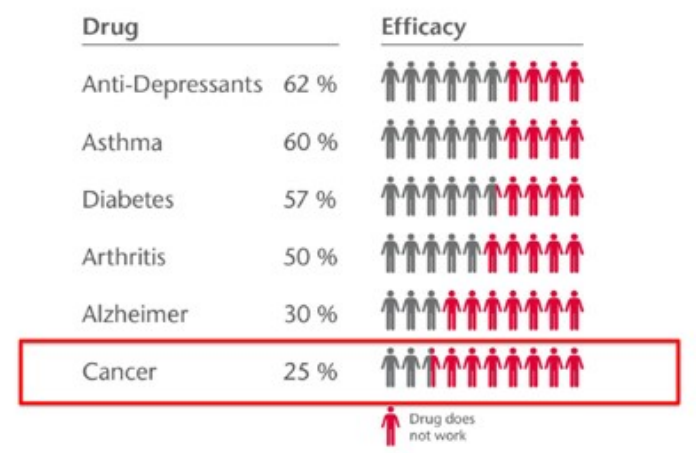

Figure 2 Drug Effectiveness

There is a lot of money spent by pharmaceutical companies and others on advertising the benefits of modern medicine. Consequently, a lot of negative information on the subject does not reach the public domain. Although modern medicine has many advantages and successes, for example, in the treatment of trauma and emergencies, it also has disadvantages and failures.

Modern medicine primarily uses surgery, radiation, and drugs to facilitate improvements in health and in the treatment of various illnesses. It is primarily involved in the treatment of the sick, unlike alternative therapies which also deal with the maintenance of health. In some cases, the therapy offered by conventional medicine is symptomatic management instead of addressing the cause of the illness. This can result in the progression of the disease as necessary lifestyle changes or corrective treatment is not initiated.

These "adverse drug reactions" are common: every year more than 2 million North Americans are hospitalized because of adverse reactions to prescription drugs. 2 The reason why drugs work in some people and cause bad reactions in others can usually be traced back to differences in genetic makeup. Medicines that work for most people may not work for someone else. They may, indeed, harm that individual. So we need two things: first, we need ways of predicting and detecting disease well before it becomes life threatening; and second, we need medicines that work for someone and their unique body.

We tend to view medical progress as some sort of continuum, along which we develop better drugs to fight whatever diseases are prevalent, better machines to image our insides and detect problems, better devices to use when joints wear out or eyes fail, better ways to treat pain or depression or loneliness; and we might be inclined to believe that the future holds more of the same. But it is not going to happen that way. Medical progress to this point has been mainly based on advances that benefit the population as a whole rather than you as an individual. Two hundred years ago, the average life span in England was only about forty years, largely because two-thirds of all children died before age four. There are statistics available suggesting that modern medicine is responsible for many deaths in America annually.

Doctors do not seek to intentionally cause harm, but the results are terrible! Especially for chronic diseases like cancer and heart disease, the treatments simply don't work. Unintended deaths are estimated at 783,000 per year, higher than all deaths in 2001 caused by heart disease $(699,697)$, and cancer $(553,251)$.

Included in this number is 106,000 deaths per year from "adverse drug reactions", or properly prescribed Drugs. That's more than 1 million deaths over a 10 year period! When combining deaths due to prescription drugs and "medical errors," the number jumps up to 7.8 million deaths.

The use of drugs in modern medicine all have sideeffects which can negatively affect one's health. There is a school of thought which advocates that modern medicine is controlled by the pharmaceutical companies. It suggests that doctors are encouraged to prescribe certain medications for the financial gain of these drug companies. 


\section{Chapter 4 - The Proposed System}

Genomic medicine is an emerging branch of medicine that involves an individual's unique genetic makeup to customize medical care. The present form of genomic medicine is a direct result of the human genome sequencing project which started in 1990 aiming to identify and map all of the human genes. Thirteen years and USD 3 billion later, the project had successfully sequenced the human genome. The year 2007 marked another turning point with the application of next generation sequencing (NGS) technology to uncover the roles of rare individual genetic variances in common diseases. The cost of genome sequencing subsequently plummeted and currently stands at around USD 1000. These technological advances have led to a major leap forward in the scope of genomics and its growing role in the delivery of healthcare.

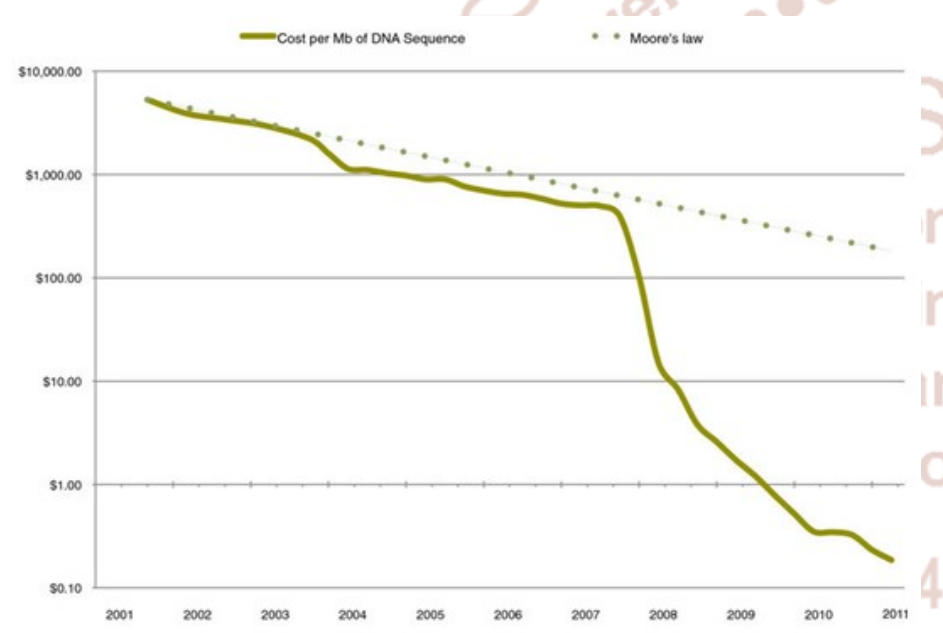

Figure 3 Cost to Sequence DNA per Mega byte

Cloud computing providers offer services that provide the infrastructure, software, and programming platforms to clients, and are accountable for the cost for development and maintenance. Compared to creating and maintaining an in-house database, cloud computing is an economical approach to genomic data management because clients pay only for the services that they need. An example of an open-source framework used to develop infrastructure for processing genomic data in a cloud computing environment is Hadoop. It breaks the data into small fragments, distributes them across many data nodes, delivers the computational code to the nodes so that they are processed in parallel, and collectively assembles the results at the end. The parallel processing of many small pieces of data, known as MapReduce, greatly shortens the computing time.

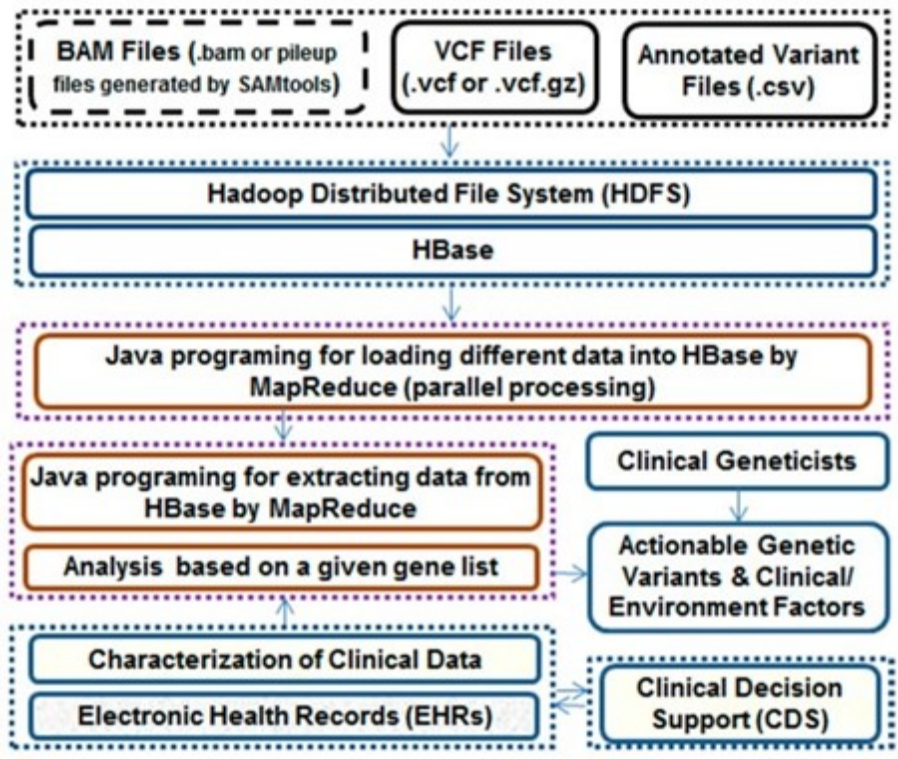

\section{Figure 4 Using Hadoop(HDFS) to process genomic data}

The Human Genome Project, completed in 2003, has provided scientists and clinicians with a diverse set of novel molecular tools that can be used to understand health and manage disease.

The use of individual genetic information plays a key role in modern medicine and will fundamentally change the way we predict, prevent, diagnose and treat diseases in the near future. for the first time in the history of medicine, health care providers as well as patients can use predictive tools to develop a new model for health care based on health planning that is proactive and preventive, as opposed to the current model in health care that is reactive, episodic, and geared toward acute crisis intervention once disease is already manifest and largely irreversible.

High-throughput genomics technology has made possible the era of precision medicine, an approach to healthcare that involves integrating a patient's genetic, lifestyle, and environmental data and then comparing these data to similar data collected for thousands of other individuals to predict illness and determine the best treatments. Precision medicine aims to tailor healthcare to patients by using clinically actionable genomic mutations to guide preventive interventions and clinical decision making. In the past 25 years, more than 4,000 Mendelian disorders have been studied at the genetic level. In addition, more than 80 million genetic variants have been uncovered in the human genome.

For people with a particular disease, using social media to compare their digital self with that of many 
others suffering from the same disorder will have major impact. By correlating the severity of disease and the effectiveness of various therapies with genomic and other "omic" data, we can expect the development of improved, individualized treatments.

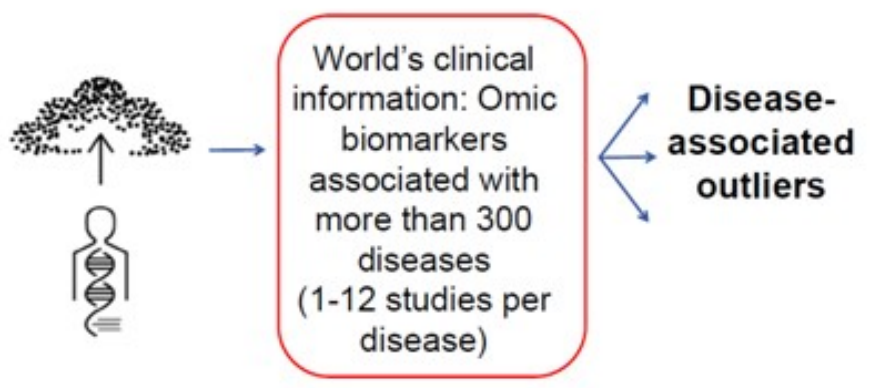

"Big data" storage and analytics

Figure 5 Uploading Genetic Information to Cloud for analysis

Early versions of the digital version of you are starting to appear, although progress has been slow because of the enormous institutional, technical, and societal issues involved. The deeply conservative instincts of the medical profession have not helped either. The first manifestation of the digital you is your electronic medical record (EMR) sometimes known as your electronic health record (EHR).

Some of the reasons for delay, it has to be admitted, are not the fault of the medical profession. Privacy is an enormous concern. Your EMR, because it is in electronic form, is susceptible to the same sort of hacking as any other personal data stored on your computer or by your credit card company or by your bank. Clearly you don't want an insurer or employer to get hold of your medical record without your authorization.

Which mutated genes are the driver mutations - the mutations that cause the cancer to grow? The cancer bioinformatics community is now tackling problems like this one with some success. However, the fact that a person's cancer may have different mutations depending on which tumor in their body is tested or from where in any given tumor the sample is taken indicates the scale of taken from different biopsies from that patient would need to be done.

If we can determine causative genes, we can then try to inhibit them. But personalized medicine is much more useful than that - a fact that will become clear as the digital you is compared with the digital versions of thousands of other people.

So how does comparison of your data with data from other individuals help identify the causes of your disorders and result in a therapy meant just for you? One example concerns the relation between genotype (your genome) and phenotype (your personal traits). Comparison of genotype with phenotype over thousands or millions of individuals will reveal in exquisite detail how various genes contribute to every aspect of you, from the color of your hair to a tendency to lisp to athletic potential. If we analyze a subset of people taking a particular drug, we can start to correlate who'll have symptoms such as dizziness, nausea, fatigue, or other nasty side effects according to their genetic makeup. The correlation between the genotype of people with a particular disease and their environment will start to bring to light the subtle relationship between environment and individual susceptibility to disease.

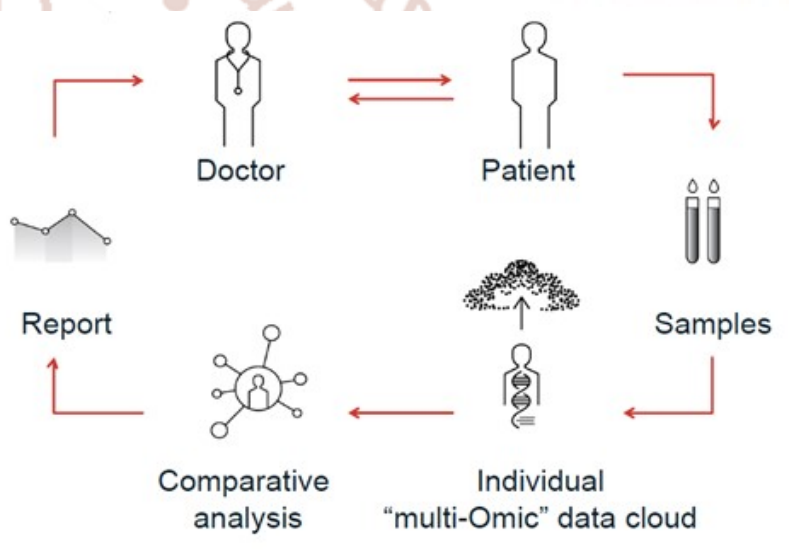

Figure 6 Proposed Diagnosis

When the pooled data were analyzed, it was found that conventional treatments actually made the condition worse, whereas other therapies were surprisingly helpful. CureTogether now has patientreported reviews of treatments for arthritis, Crohn's disease, and bipolar disorder, among other conditions. CureTogether was recently acquired by 23 andMe, a direct-to-consumer genomic sequencing and analysis company, which will gather genetic information on patients to see how this data relates to effectiveness of treatment and the toxicities associated with various drug therapies.

Genetic testing will become a cornerstone of cancer diagnosis, allowing physicians to identify and classify tumors based on their genetic signatures in addition to their location in the body. Furthermore, results from genetic testing can be useful to evaluate the prognosis 
of an individual's cancer and to cross-reference the results to known treatment options for a patient's particular mutations.

Expectations are high that genetic testing will accurately predict the risk for various diseases and eventually lead to preventive and therapeutic interventions that are targeted to at-risk individuals based on their genetic profiles.

Latest research has led to the development of Genetic Risk Scores (GRS) combining individual genetic variants associated with a specific disease. Such risk scores enable stratification of individuals into lowand high-risk groups for common disorders such as heart disease, diabetes and most cancers.

In terms of insurance, GRS for breast cancer, for example, have been shown to have a better risk prediction than a score based on non-genetic risk factors (BMI, smoking status, alcohol, and family history of breast cancer) routinely assessed in insurance underwriting. Even better predictions can, however, be achieved using a combination of genetic and non-genetic factors. Along with traditional risk factors used in insurance underwriting, reliable GRS may become an additional or alternative technique for risk stratification of insurance applicants.

Family-based analysis: Family-based NGS data enable the discovery of disease-contributing de novo mutations. Meanwhile, family-based research strategies can uncover many mutations that may be contributing to recessive, inherited as homozygous or compound heterozygous diseases. SeqHBase is a reliable and scalable computational program that manipulates genome-wide variants, functional annotations and every-site coverage, and analyzes whole genome sequencing data to identify diseasecontributing genes effectively. It is a Big Data-based toolset designed to analyze large-scale family-based sequencing data to quickly discover de novo, inherited homozygous, and/or compound heterozygous mutations.

Population-based analysis: A number of large-scale population-based sequencing studies are undergoing. For example, the PMI cohort program attempts to sequence one million or more American participants for improving our ability to preclude and cure diseases based on one's differences in genetic makeup, lifestyle, and environmental factors. By 2025, over 100 million human genomes could be sequenced. Therefore, it is critical to develop statistical toolsets on a Big Data infrastructure for analyzing the genomic data of millions of people.

Projects such as the Deciphering Developmental Disorders study, offering exome sequencing to children with severe developmental disorders, report that if a clinical exome was offered as a first line diagnostic test, $50 \%$ of these children would instantly receive a diagnosis. With advances in genomic technology, where relevant, it should be possible to identify the prime genetic cause for every rare disorder. What underpinned the success of the DDD project was the ability to match children at opposite ends of the world to each other, using a database called DECIPHER. As each child's condition was uncommon and for the doctors caring for that child, they may never have encountered a child with a similar condition before the DECIPHER database afforded the opportunity to link children with the same genetic result and phenotype. This added to the credibility that the identified variant was indeed the cause of the child's condition.

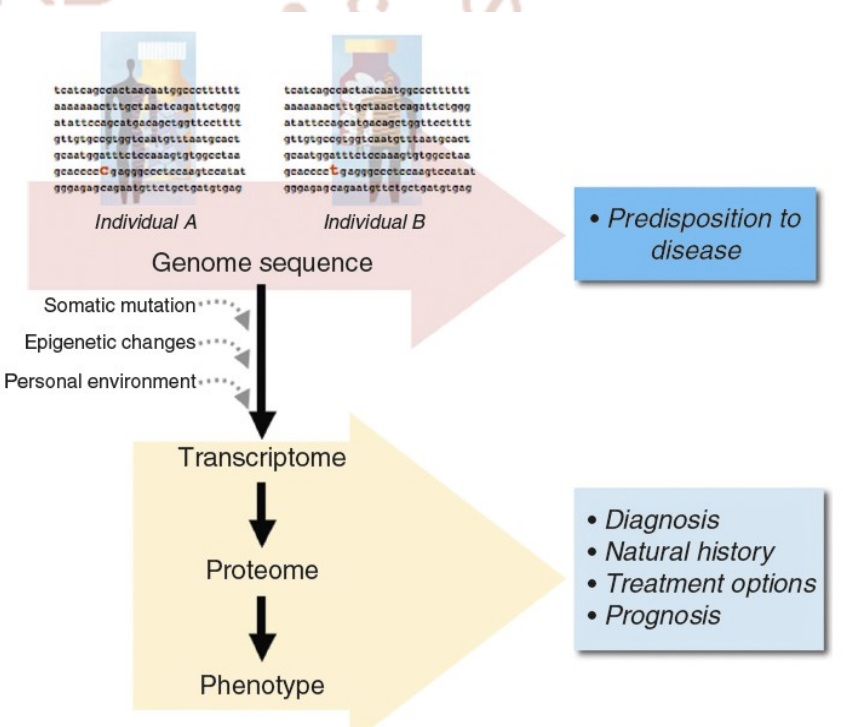

Figure 7 DNA comparision between individuals

So the digital version of you which is the sum of your genomic, proteomic, metabolomic, microbiomic, and potentially other omic data, combined with a digital record of your vital signs over time as detected by remote sensing - is on the way. This data will be informative, to say the least - particularly when you compare your digital self with the digital versions of others in your situation. The biggest single gap that remains is interpretation of this data. But as you can see from the progress being made with big data, it will become very precise, very soon. 


\section{REFERENCES:}

1) Carter T.C., He M.M. Challenges of identifying clinically actionable genetic variants for precision medicine. J. Healthc. Eng. 2016;2016

2) Vassy J.L., Korf B.R., Green R.C. How to know when physicians are ready for genomic medicine. Sci. Transl. Med. 2015

3) W. KenRedekop PhD, MPH1 Deirdre Mladsi BA2 The Faces of Personalized Medicine: A Framework for Understanding Its Meaning and Scope - Pages S4-S9, 2013

4) Genomic medicine and the NHS - planning for tomorrow today Attain.co.uk
5) Geoffrey S. Ginsburg and Huntington F. Willard, The Foundations of Genomic and Personalized Medicine 1-5

6) Dhavendra Kumar, From evidence-based medicine to genomic medicine.

7) $\mathrm{He} \mathrm{KY}$, Ge D, He MM, Big Data Analytics for Genomic Medicine, 15 Feb 2017.

8) Tonia C. Carter 1 and Max M. He, Challenges of Identifying Clinically Actionable Genetic Variants for Precision Medicine, 6 Apr 2017

9) Collins F.S., Varmus H. A new initiative on precision medicine. N. Engl. J. Med. 2015; 372:793-795

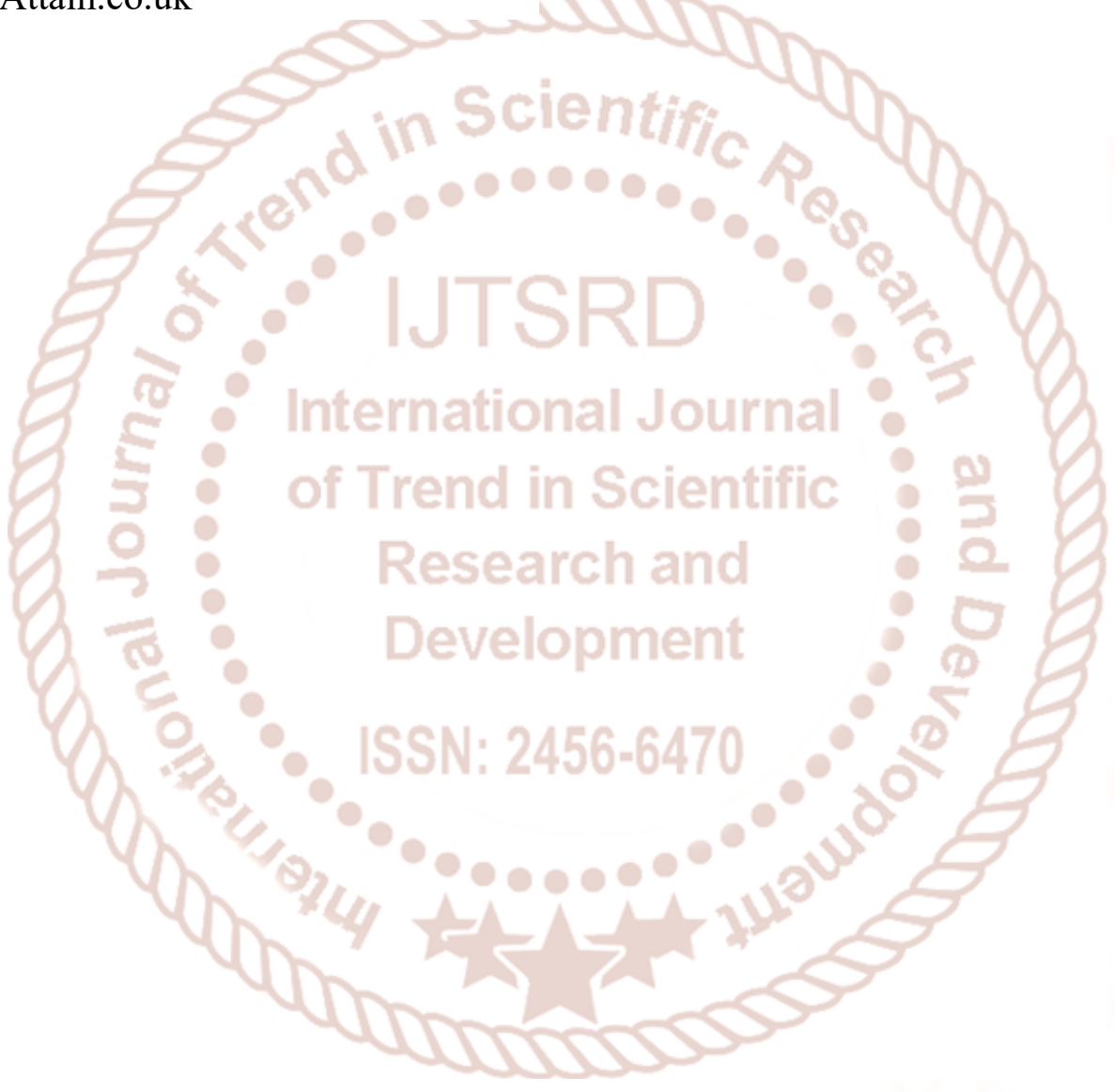

